\title{
Existence en Afrique équatoriale d'un important foyer de Dicrocoeliose bovine et ovine à Dicrocoelium Hospes (Looss, 1907)
}

\author{
par M. GRABER ef O. OUMATIE \\ Laboraloire de Farcha-Fort-Lamy Tchad
}

\begin{abstract}
RÉSUMÉ
$1^{0}$ Les auteurs font le tour de la bibliographie succincte consacrée au problème de la Dicrocoeliose bovine et ovine en Afrique noire.

Deux espèces peuvent être mises en cause : Dicrocoelium hospes (LOOSS 1907) et Dicrocoelium dendritıcum (RUDOLPHI, 1815).

La première, essentiel lement africaine, existe sûrement au Soudan, au Nigeria, au Ghana, en Guinée el au Sénégal.

Les références concernant la seconde ne sont pas très claires : elles intéressent le Ghana, le Nigeria, et, peut-être la Guinée.

$2^{\circ}$ Au cours d'enquêtes effectuées de 1954 à 1963 en Afrıque équatoriale des exemplaires de Dicrocoelium ont été recueıllis chez le zébu à Marova (NordCameroun, 1963) chez le mouton à Fianga (Tchad, 1960-63) et à Brazzaville (Congo) par le Dr ROUSSELOT sur Syncerus caffer nanus

II s'agit, dans les trois cas, de Dicrocoelium hospes. La comparaison avec $D$. dendriticum ne laisse subsister aucun doute. Les auteurs donnent une descrip. tion sommaire des deux Trématodes et une série de mensurations.
\end{abstract}

$3^{0}$ Le foyer de Dicrocoeliose décauvert est à cheval sur le Tchad et sur le Comeroun. Vers l'Ouest, il semble se rattacher au foyer Nigérien. Vers l'Est, it est lımité par la région de Fianga et, vers le Nord, il ne dépasse pas le $11^{\mathrm{e}}$ paralèle : les grandes régions d'Elevage de la République du Tchad sont, jusqu'à présent indemnes de Dicrocoeliose.

$4^{0}$ Les taux d'infestation sont élevés : 58 p. 100 pour les zébus de Maroua et 40 p. 100 pour les moulons de Fianga.

$5^{\circ}$ Chez le bøuf, la Distomatose à Fosciola gigantica est moıns fréquente que la Dicrocoeliose à Dicrocoelium hospes (36 p. 100 contre 58 p. 100). Les associations entre ces deux Trématodes touchent $27 \mathrm{p} 100$ des animaux autopsiés.

Chez le mouton, le problème se présente de la même façon, maıs Il n'y a pas d'associations entre Dicrocoelum et Fasciola.

$6^{0}$ Les modifications subies par le milieu, qu'elles soient dues au climat ou à I'homme, jouent un rôle déterminant dans les fluciuations plurıannuelles observées, dans une région donnée, entre Dicrocoelium ef Fasciola. Les auteurs citent comme exemple les varıations relevées à Fianga entre 1960 et 1963.

Une carte, deux tableaux, des figures et 32 références bibliographiques accompagnent le présent dacument. 


\section{INTRODUCTION}

La Dicrocoeliose des ruminants domestiques est une Distomatose hépato-biliaire due à la pénétration dans le parenchyme hépatique, puis dans les canaux biliaires de Trématodes appartenant à la famille des Dicrocoeliidoe ODHNER, 1910, ef au genre Dicrocoelium, DUJARDIN 1845.

Deux espèces interviennent : la première Dicrocoelium dendriticum RUDOLPHI, 1819, est cosmopolite. Elle a été signalée plus ou moins fréquemment en Europe, en Asie, en Océanie et en Amérique.

La seconde, Dicrocoelium hospes, LOOSS, 1907 semble localisée à l'Afrique noire. Décrite par LOOSS à partir d'exemplaires prélevés dans la vésicule biliaire d'un Bos Taurus $(L)$ du Soudan, elle a été sûrement retrouvée chez le bœuf au Ghana (STEWART, 1930), au Sénégal ef en Guinée chez le mouton (MOREL, 1959) et dans I'Est de la Nigeria (District d'Izi-Campbell, 1958) MONNIG (1950) confirme l'existence de ce Trématode en Nigeria et au Ghana.

Les autres références concernant les Dicrocoelium des animaux domestıques de l'Afrique de l'Ouest ne sont pas très claires. JOYEUX, GENDRE et BAER sans apporter de preuves précises, affirment que « Dicrocoelium dendriticum » est commun dans tout le continent africain et qu'il frappe le mouton et le bouf. BEAL (1929) au Ghana, CURASSON (1938) en Guinée, METTAM (1950) en Nigeria font état du même parasite. WILSON (1958), toujours dans le Nord de la Nigeria, parle de Dicrocoelium ssp.

Au cours des enquêtes menées au Tchad, au Cameroun, en R. C. A. et au Congo (Brazzaville) depuis 1954, un grand nombre de Dicrocoelium on été récoltés :

A Maroua (Nord-Cameroun, 1963-64) dans les canaux biliaires de zébus.

A Fianga (République du Tchad, 1962-63) chez le mouton.

A Bouar (R. C. A., avril-juin 1964) chez le zébu.

Le Laboratoire de Farcha possède également des exemplaires recueillis par $M$. le Dr ROUSSELOT à Brazzaville (1955) sur un Syncerus caffer nanus (Boddaert).

\section{DESCRIPTION SOMMAIRE}

Devant la confusion qui semble régner en Afrique noire sur l'identité de l'espèce en cause, il a paru intéressant de préciser les caractères différentiels des deux Dicrocoelium susceptibles d'être rencontrés. Des exemplaires frais de Dicrocoelium dendriticum provenant de moutons sacrifiés à l'abattoir, de Lyon (France) nous ont été gracieusement fournıs par $M$. le Professeur EUZÉBY que nous tenons à remercier vivement.

D'une façon générale, les Dicrocoelium sont des Distomata de taille moyenne, au corps allongé, aplati et lancéolé, plus étroit dans la région antérieure que dans la région postérieure, la largeur maximum se situanten arrıère du milieu du corps.

Ils sont transparents ef laissent voir leur organisation interne. Celle-ci comprend une ventouse antérieure terminale suivie d'un pharynx ef de deux cæcums intestinaux qui n'atteignent pas l'extrémité postérieure du corps. Au-dessous de la bifurcation des cacums, se trouvent la poche du cirre d'où l'organe fait souvent saillie, puis la ventouse postérieure.

Les testicules sont disposés obliquement l'un derrière l'autre, en arrière de la ventouse ventrale. Ils sont suivis de l'ovaire.

La région postérieure du corps au-delà de l'ovaire, est occupée par les circonvolutions utérines bourrées d'œufs. Latéralement, entre les caecums intestinaux ef la paroi du corps, prennent place les vitellogènes.

Des canaux déférents vont des testicules au pore génital.

Ce schéma est valable tant pour $D$. dendriticum que pour $D$. hospes. Pour établir une comparaison précise, des mensurations ont été effectuées sur 10 exemplaires de Dicrocoelium dendriticum et 10 exemplaires de chacun des Dicrocoelium de zébu, de mouton et de buffle, Les résultats figurent (voir tableau $n^{\circ}$ l).

Les Dicrocoelium des moutons de Fianga et des zébus de Maroua diffèrent donc sensiblement de Dicrocoelium dendriticum, dans leur largeur, dans les dimensions des ventouses, du pharynx ef des testicules et également dans la longueur des vitellogènes (fig. I et II). Par ailleurs, la lobulation des testicules de $D$. dendriticum est beaucoup plus visible que celleddes Dicrocoelium d'Afrique équatoriale. 
CARTE N* 1

REPARTITION DE DICROCCELIUM HOSPES EN AFRIQUE

- Foyers cotuellement connus

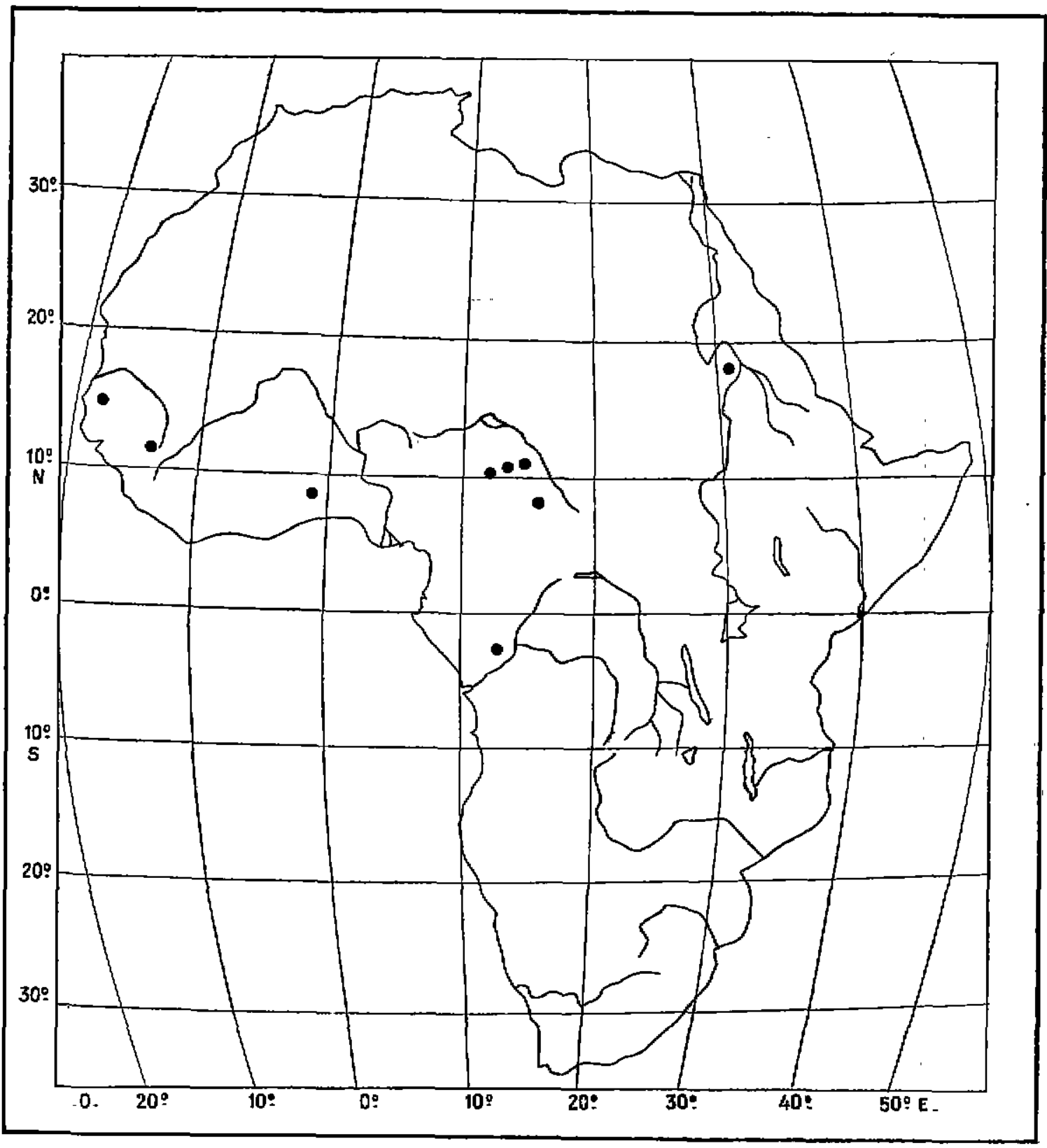


TABLEAU NO I

Mensurations

\begin{tabular}{|c|c|c|c|c|c|}
\hline \multirow[t]{2}{*}{ Organe } & \multirow[t]{2}{*}{ D.dentriticum } & \multicolumn{4}{|c|}{ Dicrocoelium hospes } \\
\hline & & Zébru & Mouton & & Bufle \\
\hline Longueur & 7,3 à 9,2 m & 7,2 aे $9,9 \mathrm{~mm}$ & 8,37 ¿ $9,08 \mathrm{~mm}$ & 6,43 & è 7,02 vall \\
\hline Largeur & 1,75 à $1,98 \mathrm{~mm}$ & 0,885 à $1,062 \mathrm{~mm}$ & 0,885 主 $1,062 \mathrm{~mm}$ & 0.507 & ì $0,944 \mathrm{zm}$ \\
\hline Ventouse antérieure & $350 \mathrm{~d} \quad 470$ & 270 \& $\quad 350$ & 295 a 350 & 260 & a 270 \\
\hline Ventouse postérieure & 425 d 470 & $350 \quad 450$ & 350 à 450 & 270 & मे 320 \\
\hline Testicula antérieur & 531 à 767 & 470 aे & 354 ì 472 & 450 & 495 \\
\hline Testicule pọstérıur & 590 à $1,06 \mathrm{~mm}$ & 450 à & 354 ̀̀ 472 & 450 & 590 \\
\hline Pheryus & $130 \mathrm{a} \quad 140$ & 94 à & 96 ̀̀ & & 97 \\
\hline Vitellogènes & 1,530 a $2,180 \mathrm{~mm}$ & 0,5 aे $1,5 \mathrm{~mm}$ & 0,944 à $\quad-1 \mathrm{~mm}$ & 531 & 香 820 \\
\hline Deufs & $36-42 \times 25-30$ & $39-42 \times 25$ & $40-42 \times 24-27$ & $36-43$ & $3 \times 25$ \\
\hline
\end{tabular}

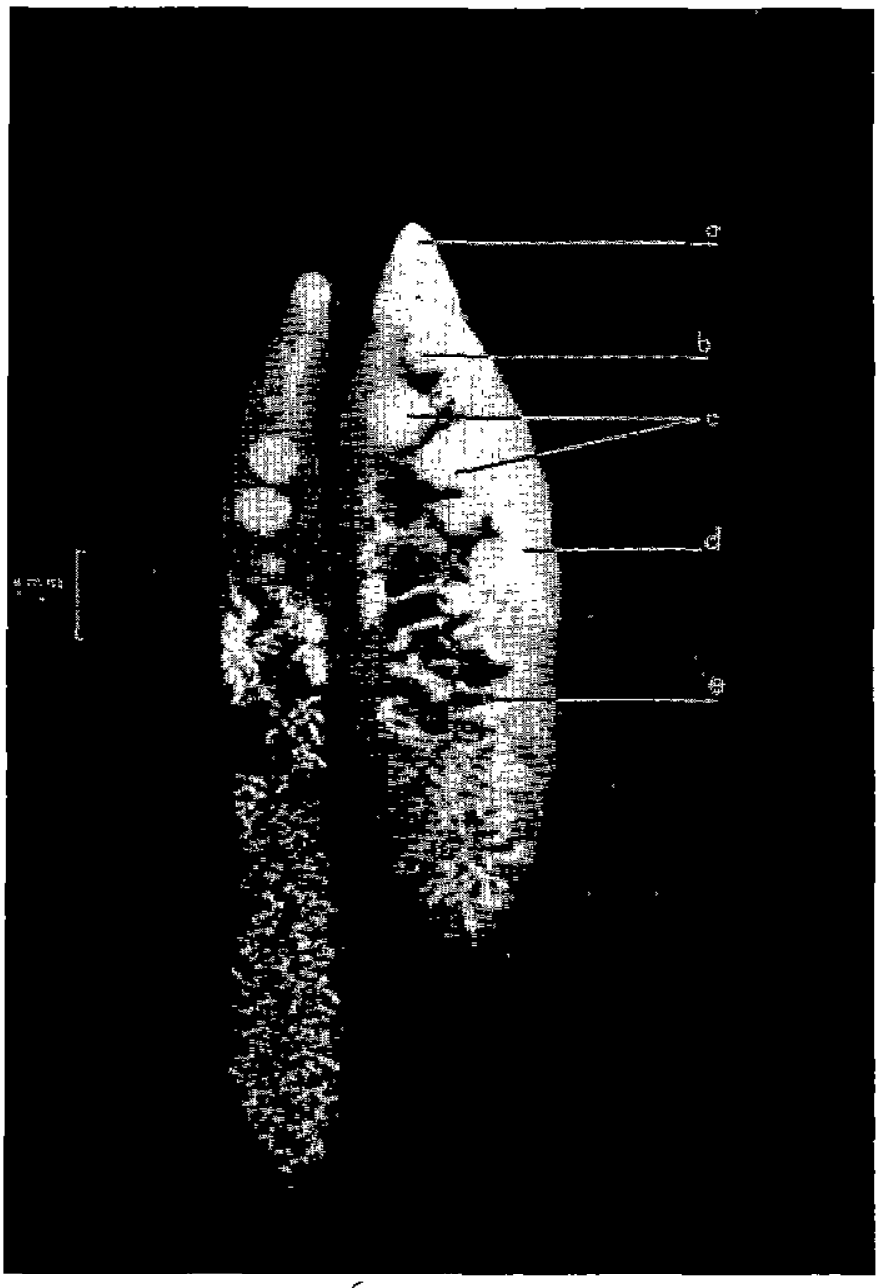

Fig. 1 : Dicroccelıum hospes (à gauche) et Dicroccelium dendriticum (à droife)
a) ventouse antérieure,
b) ventouse postérieure,
c) testicules,
d) vitellogènes,
e) utérus. 


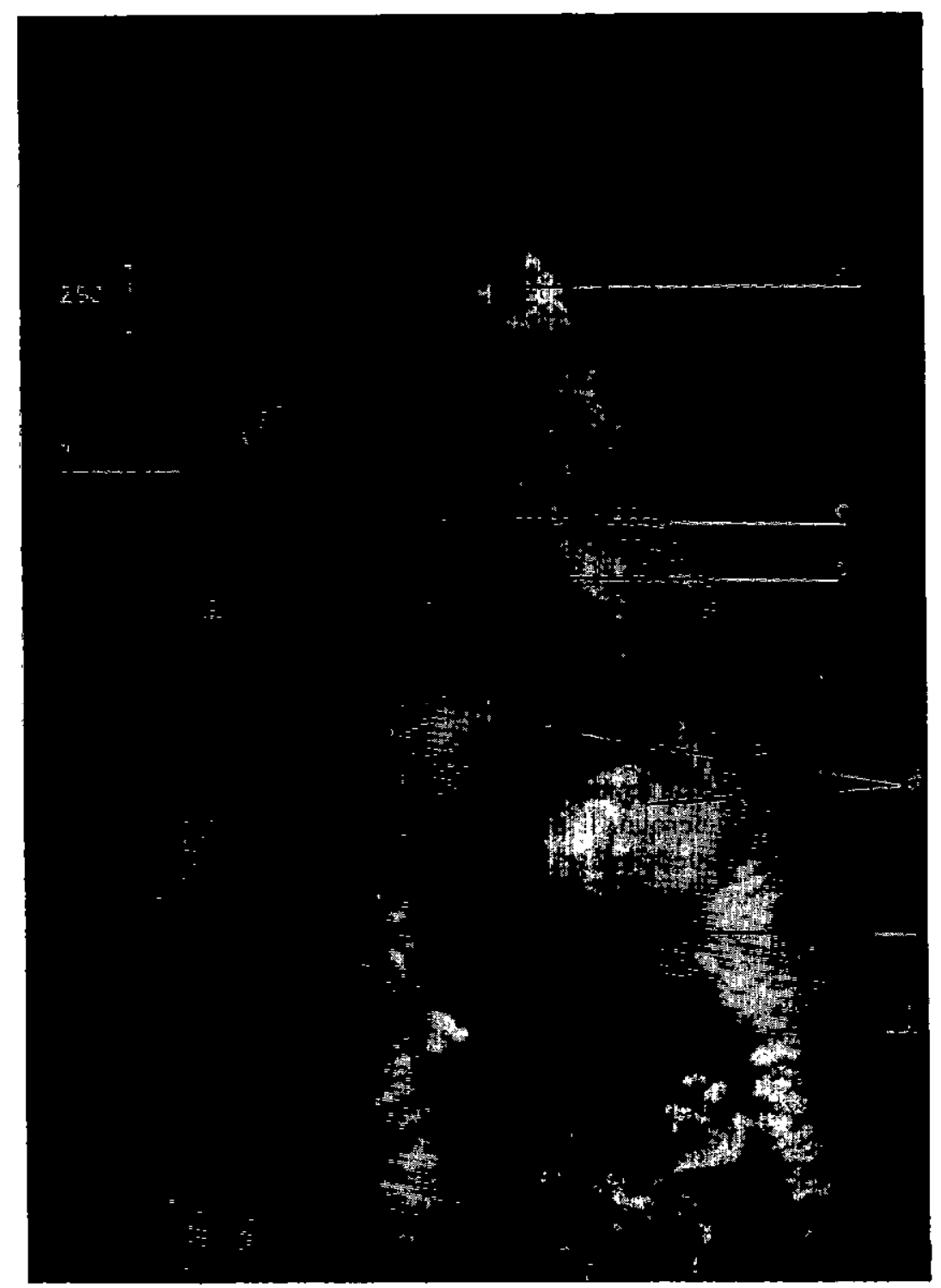

Fig. 2 : Dicroccelium hospes et Dicrocœelium dendrificum (détail)
a) ventouse antérieure.,
b) ventouse postérıeure.
c) pore génital,
d) testicules.
e) utérus,
f) vitellcgènes,
g) pharynx. 
Les Trématodes de buffle, bien que mûrs, sont ${ }^{\circ}$ de plus petite taille, et les organes moins importants, sauf le pharynx et les testicules. Aucun caractère bien net ne permet de les différemcier des Dicrocoelium du. Tchad ou du Cameroun.

Si l'on compare avec la description originale de LOOSS (1907 a) reprise par NEVEU-LEMAIRE (1936), TRAVASSOS (1944) et SKJABIN (1952), il s'agit bien de Dicrocoelium hospes.

Dans la littérature, le parasite n'est pas complètement inconnu, puisqu'il a été cité à l'occasion de divers travaux comparatifs ou non (TRAVASSOS, 1918, 1919 ; DOLLFUS, 1922 ; NICOLL, 1923 ; NORTHUP, 1928 ; SANDGROUND, 1929, MACY, 1932 ; CAMERON, 1934 ; GOHAR, 1935; MC INTOSH ef MC INTOSH, 1935 ; YAMAGUTI, 1958).

\section{RENSEIGNEMENTS COMPLÉMENTAIRES}

\section{a) Répartition géographique.}

Le foyer Oubanguien étant encore mal délimité, il ne sera question ici que du foyer le plus important, celur du Tchad-Cameroun. Il paraît actuellement inscrit dans un quadrilatère dont les sommets sercient constitués par les marchés de Dourom et de Papata et les agglomérations de Yagoua et de Fianga (carte $n^{\circ}$ II). La ville de Maroua où ont eu lieu la plupart des autopsies est incluse dans le périmètre contaminé, ainsi que les gros marchés à bestiaux de Moulvoudai, Malinabata ef Bogo. La zone atteinte ne dépasse pas jusqu'à présent les lacs Toubouris à l'Est et le 11 e parallèle au Nord. Les grandes régions d'Elevage du Tchad ne semblent pas touchées en dix ans sur 4.650 bovins et plus de 4.000 moutons autopsiés, Il n'a pas été possible de mettre en évidence $D$. hospes.

II n'en est pas de même vers I'Ouest, c'est-àdire vers la Nigeria où la Dicrocoeliose existe dans l'Est de ce pays (district d'lzy). Le foyer camerounais représente-t-il la branche extrême du foyer Nigérien ? Actuellement, Il est Impossible de l'affirmer avec certitude.

Dans les deux cos, district d'lzy et circonscription de Yagoua, il s'agit de régions s'adonnant à la riziculture et les rizières, après la récolte sont pâturées par le bétall.

\section{b) Fréquence de l'infestation.}

A Maroua sur les 140 zébus autopsiés en août 1963, 81 d'entre eux hébergeaient Dicrocoellum hospes, solt 57,8 ₹. 100. Ce taüx d'infestation est impressionnant. A. l'échelle mondiale, it représente vraisemblablement un record.

A Fianga, le nombre de moutons parasités est moins élevé : environ 40 p. 100 en 1963.

A Bovar, les premiers résultat's de l'enquête donnent un taux de 3,5 p. 100 chez les bouvillons.

c) Coexistence chez un même animal de la Distomatose à Fosciola gigantica et de la Dicrocoeliose à Dicrocoelium hospes.

En milieu tempéré ef avec Dicrocoelium dendriticum chez le mouton, on estime (EUZÉBY, 1958) que la petife Douve est moins fréquente que la grande Douve et que les deux espèces sont rarement associées entre elles, du falt de la nonconcidence de leurs aires géographiques qui se trouvent sous la dépendance étroite des mollusques hôtes Intermédiaires.

Au Cameroun, chez le bœuf, il n'en est pas tout à fait ainsi : $D$. hospes est plus abondant que F. gigantica (57, 8 p. 100 contre 36 p. 100) et les deux Trématodes cohabitent dans les canaux biliaires du même animal dans 20 p. 100 des cas environ.

A Fianga, les cas, de Dicrocoeljose ovine sont deux fois plus nombreux que les cas de Distomatose (1963), mais il n'existe pas d'association entre les deux Helminthes.

Les hôtes intermédiaires de $D$ : dendriticum et de $F$. hepatico dans une zone atteinte par ces deux parasites, sont donci capables de s'adapter à des conditions de vie qui sont quelquefols très différentes de celles qui leur conviendraient le mieux (EUZÉBY, 1958), Cette constdtation semble également valable pour $D$. hospes et $F$. gigantica au Nord-Cameroun.

d) Fluctuation pluriannuelle du parasitisme par Fasciola gigantica ef Dicrocoelium hospes.

La proportion relative entre les deux espèces esł sujette à des variations qui tiennent au climat et aux modifications subies par le milieu.

En juin 1960, les lacs Toubouris sont envahis par de nombreux Nymphoeo et le niveau des eaux est très haut. Un grand nombre de Iımnées ont pu être recueillies sans difficulté. 


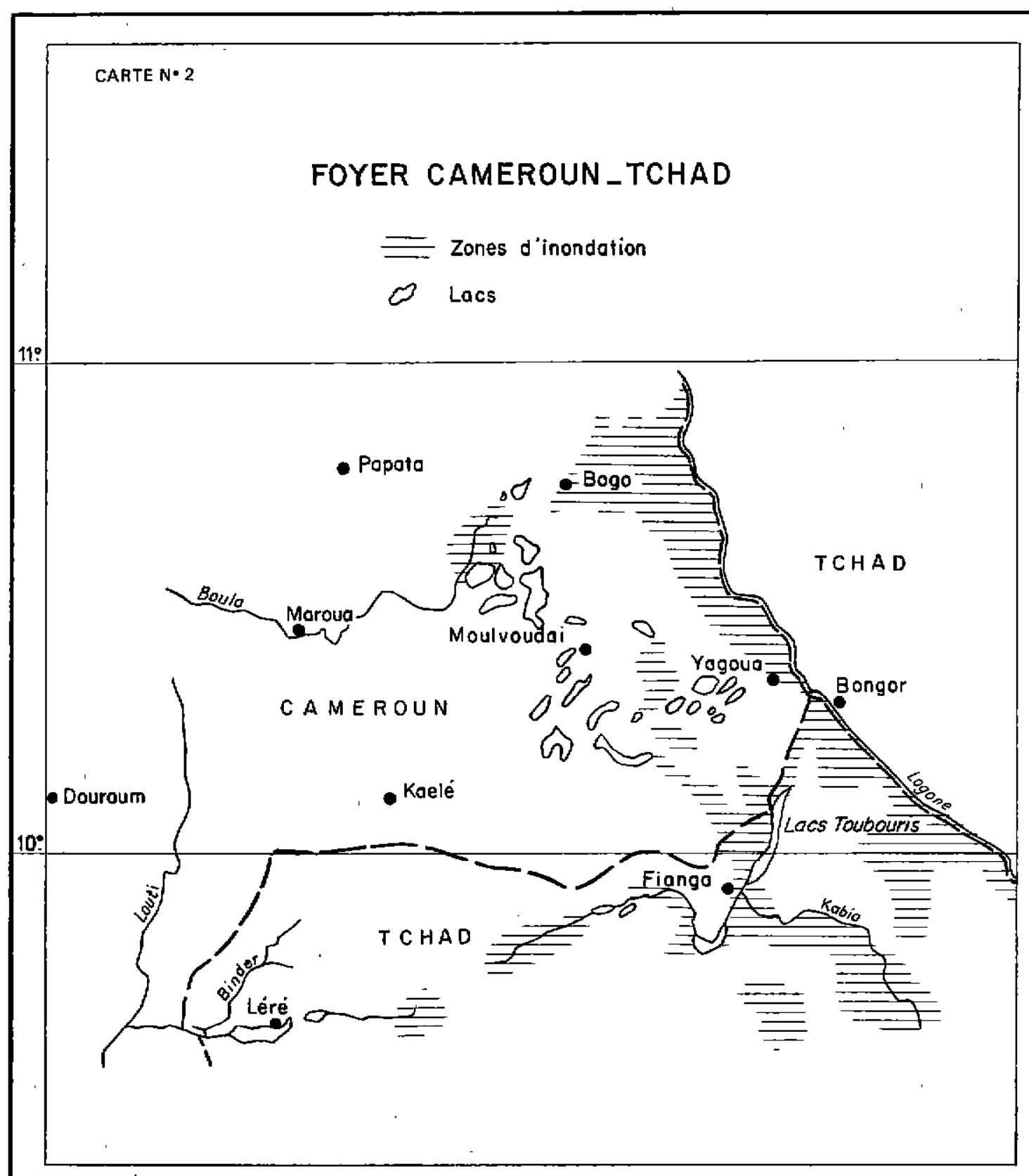




\begin{tabular}{|c|c|c|c|c|}
\hline Années & $\begin{array}{c}\text { Nombre de moutons } \\
\text { cutopsiés }\end{array}$ & Epoque & $\begin{array}{c}\text { Nombre de moutons } \\
\text { infestés par } \\
\text { F.gigantica }\end{array}$ & $\begin{array}{c}\text { Nombre de moutons } \\
\text { Infestés par } \\
\text { D. hospes }\end{array}$ \\
\hline 1960 & 14 & Juin & 13 & 0 \\
1962 & 24 & Juin & 6 & 1 \\
1963 & 22 & Mai & 4 & 9 \\
\hline
\end{tabular}

En juin 1962, les eaux sont basses, laissant à découvert entre les deux lacs un vaste fond non marécageux où les animaux vont pâturer. Les Nymphaea ont disparu. Les rives des lacs, sur une distance de $1,50 \mathrm{~m}$ environ, sont tapissées de graminées et de joncs, formant un feutrage très épais. Plus haut, le débolsement est intense. Les limnées sont rares.

En mai 1963, le milıeu n'a pas changé par rapport à l'année précédente. Les récoltes de limnées sont toujours aussı médıocres. Par contre, des mollusques terricales sont découverts en abondance. L'interprétation de ces observations permet de penser :

$1^{0}$ Qu'à f'origine, seule la Distomatose à Fasciola gigantica est fortement implantée dans le pays.

$2^{\circ}$ Les années suivantes, sans doute à la suite de l'introduction de moutons porteurs de $D$. hospes venus du foyer camerounais voisin, la Dicrocoelıose ovine fait une timide apparition.

3o Parallèlement, la Distomatose diminue Plusieurs rassons peuvent être ınvoquées : années sèches, baisse du niveau des eaux, avec comme conséquence destruction d'un assez grand nombre de mollusques aquatiques, ainsi qu'en témoigne la forte proportion de coquilles vides ramassées sur les bords, présence sur les rives d'un milieu impropre à la survie des hôtes intermédıaires et disparition des Nymphaea qui servent de supports au Bulıns et aux Limnées (GRETILLAT, 1963), déboisement dû à la main de l'homme.

Dans ce contexte plus sec, les conditions de développement de $D$. hospes deviennent favora bles. Comme dans le cas de D. dendriticum (KRULL et MAPES, 1952-1953), elles pourraient dépendre de deux types d'hôtes intermédıaires: des mollusques terricoles bien adaptés à la sécheresse et des fourmis, la preuve reste d̀ faire.

Les modifications subies par le milieu, qu'elles tiennent au clımat ou à l'homme, semblent être à l'origıne des fluctuations pluriannuelles observées dans une région donnée entre Dicrocoelium et Fasciolo. Le même fait a déjà été signalé en France dans le département de la Côte-d'Or (EUZÉBY, 1958).

\section{CONCLUSIONS}

$1^{\circ}$ Au cours d'enquêtes effectuées de 1954 à 1964 en Afrique Equatoriale, des exemplaires de Dicrocoelium ont été recuellis chez le zébu à Maroua (Nord-Cameraun 1963) et d̀ Bouar (R. C. A. 1964), chez le mouton à Fianga (Tchad, 1962-1963) et à Brazzaville (Congo), par le Dr ROUSSELOT, sur Syncerus caffer nanus.

Dans les quatre cas, il s'agit de Dicrocoelium hospes (LOOSS, 1907). La comparaison avec Dicrocoelium dendriticum ne laisse subsister aucun doute. Les auteurs donnent une description sommaire des deux Trématodes ef une série de mensurations.

$2^{\circ}$ Le plus important foyer de Dicrocoeliose est à cheval sur le Tchad et sur le Cameroun. Vers l'Ouest, il semble se rattacher au foyer Nigérien. Vers l'Est ił est limité par la région de Fianga ef les lacs Toubouris. Vers le Nord, Il ne dépasse pas le $11^{\mathrm{e}}$ parailèle : les régions d'Elevage de la République du Tchad, sont, jusqu'à présent, indemnes de Dicrocoeliose.

30 Les taux d'infestation sont élevés : 58 p. 100 pour les zébus de Maroua ef 40 p. 100 pour les moutons de Fianga.

4o Chez le bouf, la Distomatose à Fosciola gigon- 
tica est moins fréquente que la Dicrocoeliose à Dicrocoelium hospes (36 p. 1.00 contre 58 p. 100). Les associations entre ces deux Trématodes touchent 20 p. 100 des animaux autopsiés.

Chez le mouton, le problème se présente de la même façon, mais il n'y a pas association entre Dicrocoelium et Fosciola. $5^{\circ}$ Les modifications subies par le milieu, qu'elles soient dues au climat ou à l'homme, jovent un rôle déterminant dans les fluctuations pluriannuelles observées, dans une région donnée, entre Dicrocoelium et Fasciolo. Les auteurs citent comme exemple les varıations relevées à Fianga entre 1960 et 1963.

\section{SUMMARY}

10 The authors have made a survey of the brief bibliography relating to the problem of Bovine and Ovine Dicrocoeltum infection in Africa.

Two species are incriminated : Dicracoelium hospes (LOOSS, 1907) and Dicrocoelium dendriticum (RUDOLPHI, 1803). The first, essentially African, exists certainly in Sudan, Nigeria, Ghana, Guinea and Senegal.

The references relating to the second are nol very definite: they refer to Ghana, Nigeria and perhaps to Guinea.

$2^{\circ}$ During investigations carried out from 1954 to 1963 in equatoria Africa, specimens of Dicrocoelium hove been collecled from Zebu cattle at Marova (North Cameroun, 1963) and at Brazzaville (Congo) by Dr ROUSSELOT" from Syncerus caffer namas. In all three cases, the parasite was identified as D. hospes comparison with specimens of $D$. dendriticum leaves no doubt. The authors give a concise description of the two Tremalodes and a series of measurements.

$3^{0}$ The nucleus of Dicrocoelium infection rests between Chad and Cameroun. Towards the West, it appears to be connected with another infected area in Nigeria. Eastward, it is limited to the region of Fianga and to the North no infection is found above, the 11 th parallel: The stock raisıng regions of the Republic of Chad are, up till the present time, unfouched by Dicrocoelium.

40 The number of animals infected is high, being 58 per 100 among Zebus of Maroua and 40 per 100 of the sheeps in Fianga.

$5^{\circ}$ Fasciolasis (Fasciola gigantıca) in cattle is less frequent than Dicrocoelium infection (D. hospes) the rate of occurence being 36 per 100 against 58 per 100 . 27 per 100 of the animals autopsied contained both these Trematodes.

In the sheep, the position is much the same except that the two parasites are not found together in the same animal.

$6^{\circ}$ The modifications of environment whether due to climate or to man, play a part in determining the fluctuations observed over several years in a given region between Dicrocoelium and Fasciola. The authors mention as example the variations observed in Fanga belween 1960 and 1963.

One map, two tables, diagrams and 32 references accompany this paper.

\section{RESUMEN}

\section{Existencia en Africa ecuatorial de un centro importante de Dicrocoeliosis bovina y ovina con Dicrocoelium Hospes}

10 Los autores estudian la bibliografia sucinta consagrada al problema de la Dicrocoeliosis bovina y ovina en Africa negra.

Se acusan dos especies : Dicrocoelium hospes (LOOS5, 1907) y Dicrocoelium dendriticum (RUDOLPHI, 1815).

La primera, esencialmente africana, existe seguramente en el Sudan, Nigeria, Ghana, Guinea y Senegal. 
Las referencias de la segunda no son muy claras : Interesan Ghana, Nigeria y, tal vez, Guinea.

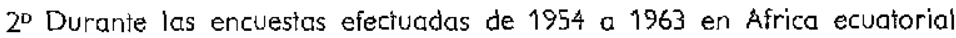
ejemplares de Dicrocoelium fueron recogidos en el cebú en Marua (Norte-Comeron, 1963) en la oveja en Fianga (Tchad, 1960-63), y en Brazzaville (Congo) por el Dr ROUSSELOT en Syncerus coffer nonus.

Se trata, en los tres casos, de Dicrocoelium hospes. La comparación con D. dendrificum no deja subsistir ninguna duda. Los autores describen someramente los dos tremátodos y una serie de medidas.

$3^{\circ} \mathrm{El}$ centro de Dicracoeliosis descubierto se encuentra a caballo en el Tchad y en el Cameron Hacia el Oeste, parece junlarse con el centro Nigeriano. Hacia el Este, se limita par la región de Fianga $y$, hacia el Norte, no pasa el paralelo undecımo: las regiones de crianza de la Republica del Tchad son, hasta ahora, indemnes en cuanto a la Dicrocoeliosis.

$4^{\circ}$ Los terminos medios de infección son elevados : 58 por 100 en los cebús de Marua y 40 por 100 en las ovejas de Fianga.

$5^{\circ}$ En el buey, la Distomatosis con Fasciolo gigantica es menos frecuente que la Dicrocoeliosis con Dicrocoelium hospes (36 por 100 contra 58 por 100). Las asociaciones entre estos dos tremátodos atacan 27 por 100 de los animales autopsiados.

En la oveja, se presenta el problema de la misma manera, pero no hay asociaciones entre Dicrocoelium y Fasciola.

$6^{\circ}$ Las modificaciones occuridas en el medio, yo sean debidas al clima o al hombre, desempenan un papel determinante en las fluctuaciones piurianuales observadas, en una región determinada, entre Dicracoelium y Fasciala. Los autores citan como ejempla las variaciones en Fianga de 1960 a 1963.

Un mapa, dos cuadros, figuras y 32 referencias bibliograficas adjuntas al presente documento.

\section{BIBLIOGRAPHIE}

1. ANTIPIN (D. N.), ERSHOV (V. S.), ZOLOTAREY (N. A.) et SALYAEV (V. A.) (1956). Parasitology and parasitic diseases of lives* tock. State publishing house for agricultural literature Moscou - israel program for scientific translations, 1960, $523 \mathrm{pp}$.

2. BEAL (W. P. B.) (1929). - Rep. Vet. Dept. Accro, 8.

3. CAMERON (T. W.M.) (1951), - The internal parasites of domestic animals: A manual for veterinory surgeons. 161, fig. 97 a.

4. CAMPBELL (A. D.) (1958). - Ann. Rep. Vet. Dept. 1957-58, East Nigeria, Enugu, 8.

5. CHANDLER (A. C.) (1956). - Introduction to parasitology. New York, 305.

6. CURASSON (G.) (1938). - Rapport sur le fonctionnement du Service Zootechnique et des Epizooties de l'A. O. F. pendant l'année 1935. Bull. Off.' Int. Epiz. 15, 9/10, 870-89.

7. DAWES (B.) (1946). - The Trematoda. Gambridge $644 \mathrm{pp}$.
8. DOLLFUS (R.) (1922). - Variations de la forme du corps, la position ef la forme des testicules chez Dicrocoelium lanceolatum (RUDOLPHI). Bull. Soc. Zool. France, 47, 334.

9. EUZEBY (J.) (1958). - Lo Dicrocoeliose des ovins. Bull. Off. Int. Epiz., 50, 1, 356-74.

10. GRETILLAT (S.) (1961). - Prophylaxie antibilharzienne et antidistomienne-Monographie. Inst. Elev. Med. Vet. Trop. Dakar, 25 pp.

11. GOHAR (N.) (1935), — Liste des Trématodes et de leurs $h$ ôfes invertébrés signalés dans la vallée du Nil. Ann. Parosit. Hum. Comp., $13,1,87$.

12. JOYEUX (C.), GENDRE (E.) et BAER (J.) (1928). - Recherches sur les helminthes d'A. O. F. Monographie. Coll. Soc. Path. Exot. Paris, 45 pp.

13. KRULL (W. H.) et MAPES (C. R.) (1952-53). - Studies on the biology of Dicrocolium dentriticum Rudolphi, 1819-Looss, 1899 (Trematoda : Dicrocoeliidae) including its relation to the intermediate host Cionella lubrica 
(Muller). Corn. Vet., 41, 382-444 ; 42, 253-77 ; $339-351,464-484,603-4 ; 43199,202 ; 389-$ 410.

14. LAPAGE (G.) (1956). - Veterinary parasitology, Edinburgh., 248-49.

15. LOOSS (A.) (1907 a). - Notizen zur helminthologie Aegyptens. VII Ueber einige neve trematoden der eagyptischen fauna. Centralbl. Bakt. Orig. 43 478-90.

16. LOOSS (A.) (1907 b). - Ueber einige zum Teil neve Distomen enropaeischen fauna. Centrolbl. Bokt. Orig., 43, 604-13.

17. MACY (R. W.) (1931). - New bat trematoda of the genera plagiorchis, limatulum ef Dicro. coelium. J. Porasil., 18, 28-33.

18. MC INTOSH (A.) et MC INTOSH (D.) (1935). - Additional notes on two bat parasites, Dicrocolium lasiuri Mc Intosh, 1933 (Nematoda, Dicrocœliidae) and litomosa americana Me Intosh, 1932 (Nematoda, Filariidae). Proc. Helm. Soc. Wash. 2, 60-3.

19. METTAM (R. W. M.) (1950). - Ann. Rep. Vet. Dept. year 1948. Lagos, Nigeria, 74.

20. MÜNNIG (H. O.) (1950). - Veterinary helminthology and Entomology. London 38.

21. MOREL (P.) (1959). - Les Helminthes des animaux domestiques de l'Afrique Occidentale. Rev. Elev. Med. Vet. Pays. Trop., 12, 2 , 153-174.

22. NEVEU-LEMAIRE (M.) (1936). - Traité d'Helminthologie médicale ef vétérinaire. VigotParis, 106
23. NICOLL (W.) (1923). - A reference list of the Trematode Parasites of British mammals. Parasit., 15, 236-52.

24. NORTHUP (F. E.) (1928). - notes on some Trématodes from bats. J. Burmo Res. Soc. Rangoon, 18, 80-97.

25. SANDGROUND (J. H.) (1929). - A nex liver fluke from a monkey and new parasilic roundworms from various african mammals. Proc. U. S. Not. Mus. 75 (12), 1-11.

26. SKRJABIN (K. I.) (1952). - Principes of Trematodology. Vol. VII. Cephaloporidae Monodhelmidae, Dicrocoelidae, Gorgoderidae. Ed. Acod. Sci. U.R.S.S., 762 pp, 263 figs.

27. STEWART (J. L.) (1930). - Rep. Vet. Dept. yoer 1929-30 - Accra - 12.

28. TRAVASSOS (L.) (1918), - Helmintos parasitos de animals domesticos. I Dicrocoelidae. Rev. de Vet. e. Zoot., 8, 1, 3, 15.

29. TRAVASSOS (L.) (1919). - Contribuiçao para e sistematica dos Dicrocoelinae Looss, 1899. Arq. Esc. Sup. Agric. Med. Vet., 3, 7-24.

30. TRAVASSOS (L.) (1944), - Revisao da familia Dicroecoeliidae Odhner, 1910. Monogr. Inst. Oswaldo Cruz., no 2, 357 pp. 124 pl.

31. WILSON (S. G.) (1958). - Ann. Rep. Dept. Vet. Serv. Northern Nigerio-Kaduna, 15.

32. YAMAGUTI (S.) (1958). - Systema Helminthum. Vol I. The digenetic Trematodes of Vertebrates. New York Intersci : Pub., Part 1,979 pp. ; Part II, 981-1575 